\title{
Localization of Broken Surgical Bur in the Submandibular Space: Its Prevention, Retrieval and the Role of Cone Beam Computed Tomography (CBCT)
}

\author{
Vimal Kalia*, Geeta Kalra, Gulzar Singh and Vikas Sharma
}

Department of Oral and Maxillofacial Surgery BRS Dental College and Hospital, India

\begin{abstract}
Surgical removal of a broken instrument can be a difficult procedure due to proximity of vital anatomical structures of the region. In the mandibular posterior lingual region the difficult access to the area adds to this difficulty. The main concern in such cases is localization of the foreign body. Here we present a case report of such a case, with its complete management and a careful technique of retrieval. The main aim of this study was to highlight the use of CBCT in such cases.
\end{abstract}

Keywords: Mandibular; Maxillofacial; Bur fatigue

\section{Introduction}

Removal of impacted mandibular third molars is the most common surgical procedure performed in dental surgery and the usage of surgical burs, both flat fissure and tapering fissure are a part of the armamentarium. Burs in clinical practice may be subjected to considerable wear and subsequently breakage. Improper technique, reusage of a bone cutting bur, bur fatigue and weakness of the alloy or the combinations of the above can sometimes lead to a broken bur in the surgical area during oral and maxillofacial surgical procedures. The incidence of a broken bur is fairly uncommon and can be prevented by following surgical protocols, using sweeping strokes rather than forcing the bur into the bone and replacing the burs regularly in the surgical kit.

Yet, when such a complication occurs irrespective of the cause, the main concern is the localization of the broken instrument and its retrieval. The removal of the broken instrument may be simple if the broken end is visible and accessible but this is not always the case. In such cases where the broken bur is not visible, locating and surgically exploring the broken instrument (bur) is difficult and time consuming. Besides the patient is alarmed and uncomfortable with the awareness of a foreign body in his jaw.

Here, a technique utilizing a Cone Beam Computed Tomography (CBCT) to locate the exact position of the bur and its surgical retrieval is described. An informed consent of the patient has been taken before publication of this study and same has been approved by ethics committee.

\section{Case Report}

A 25 year old male was referred to the maxillofacial centre with the complaint of pain and limitation in mouth opening. History

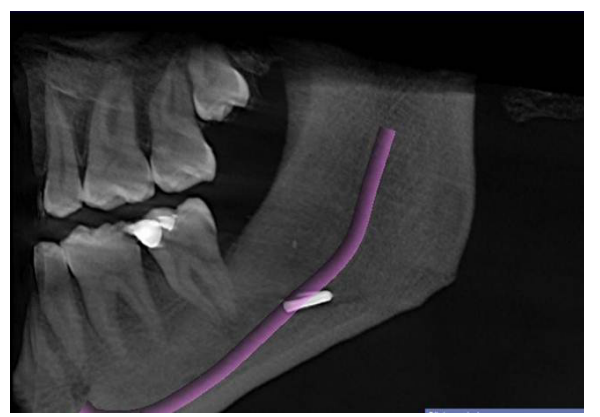

Figure 1: $А$ СВСТ image showing proximity of the broken bur to nerve. revealed that two days ago the patient had undergone a removal of impacted 38 tooth which was an extensively long procedure and the tooth was removed in fragments. The operator did notice that during the procedure the bone bur had broken and the head of the bur was lost. An attempt was made to locate the bur for removal but it failed. IOPA radiograph revealed the broken bur fragment beyond the tooth socket which appeared empty and the impacted tooth raminants were not present.

On clinical examination, a slight extraoral swelling was noticed and intraorally a sutured, healing wound was seen. With the ensuing inability to open the mouth only a limited palpation was possible as aggressive palpation could have displaced the broken bur further. The patient did not complain of any dysaesthesia of the lip or tongue. It was also not clear whether the fragment was within the socket, embedded in the socket walls, in the buccal or lingual plate or was outside the socket.

Hence, it was decided to order a CBCT mandible to exactly ascertain the location of the foreign body. The CBCT revealed the position of the fragment some distance below the socket and it was evident that it had perforated the lingual cortex at the base of the socket and was lying in the sub-mandibular space close to the mylohyoid ridge (Figures 1 and 2).

After a week, the wound had healed and the mouth opening had improved (Figure 3). Under local anesthesia (Articaine HCL with 1:100,000 adrenaline) a lingual incision was made distal to the 37 tooth and extended to the ascending ramus. Incision was extended into the lingual gingival crevix of 36 tooth. Lingual mucoperiosteal flap was raised very slowly and carefully from the anterior aspect proceeding posteriorly and inferiorly. As the submandibular space was visible, the bur end could be seen just below the base of the socket. A curved haemostat was used to hold the fragment which was then removed (Figure 4). After copious irrigation the surgical site was closed with $3 / 0$ vicryl (Figure 5). Post-operative healing was uneventful on one week follow up.

*Corresponding author: Vimal Kalia, Department of oral and maxillofacial surgery, BRS Dental College and Hospital, India, Tel: 09911029998; E-mail: vimal@kalia.in

Received September 23, 2015; Accepted November 13, 2015; Published November 20, 2015

Citation: Kalia V, Kalra G, Singh G, Sharma V (2015) Localization of Broken Surgical Bur in the Submandibular Space: Its Prevention, Retrieval and the Role of Cone Beam Computed Tomography (CBCT). J Clin Case Rep 5: 636. doi:10.4172/2165-7920.1000636

Copyright: ( $) 2015$ Kalia V, et al. This is an open-access article distributed under the terms of the Creative Commons Attribution License, which permits unrestricted use, distribution, and reproduction in any medium, provided the original author and source are credited. 


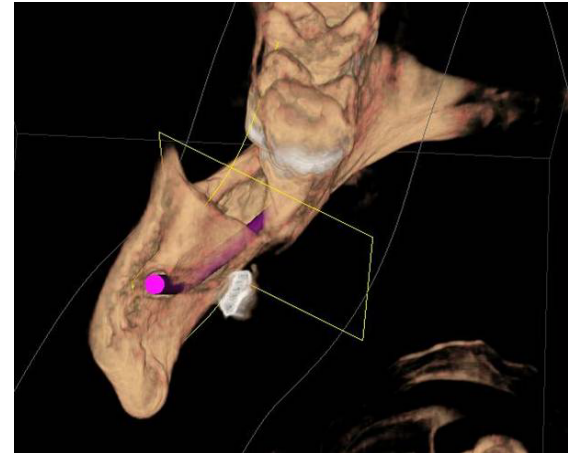

Figure 2: $\mathrm{CBCT}$ section showing presence of bur in submandibular space.

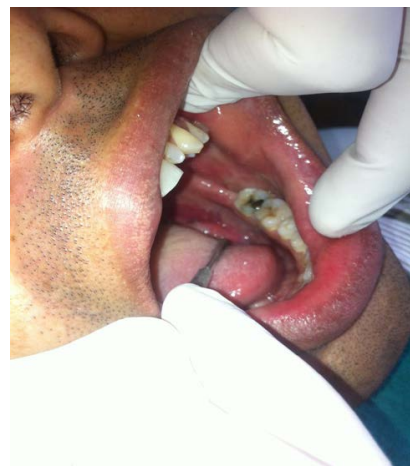

Figure 3: The preoperative clinical view.

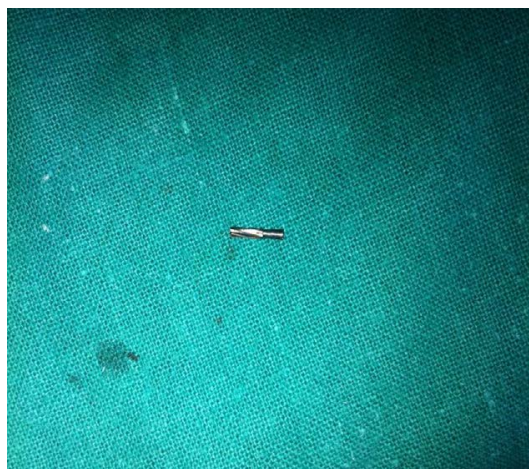

Figure 4: The broken and retrieved bur.

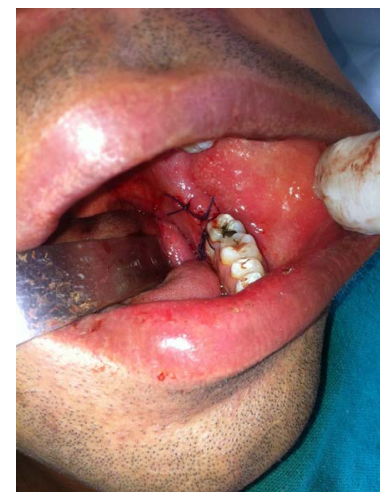

Figure 5: The postoperative clinical view.

\section{Discussion}

If an instrument (bur) breakage occurs, it may present as two clinical situations:

a) It maybe in the form of a visible fragment which can easily be then grasped with a hemostat and pulled out.

b) It may be an embedded fragment and in this situation may not be visible in the surgical area due to hemorrhage or it may have been displaced further like in this case where the bur fragment was partially located in the sub-mandibular space after perforating the lingual plate.

Usually attempts are made to palpate the mucosa (in this case the lingual mucosa) to feel the broken instrument which may further displace the instrument fragment into the deeper free space and retrieval becomes complicated. It is important to inform the patient of the broken fragment, calm the patient and immobilize the jaw and refer him to an oral and maxillofacial surgeon immediately.

Apart from a thorough clinical examination, examination of the surgical wound is essential. The initial radiograph may reveal the presence of the instrument fragment. The literature describes use of CT scan to locate the exact position of the foreign body [1-4]. Cone beam CT if available, can provide the added advantage of low radiation exposure and 3 dimensional view $[5,6]$. Also 3D reconstruction which can be rotated to predict the exact position of the foreign body with precision helps to plan a surgery for retrieval with predictable outcome. It helps to measure the exact location of the fragment from the alveolar socket as well as anatomical landmarks so that the surgeon can proceed with an image guided surgical exploration and retrieve the fragment.

Various authors recommend a waiting period before retrieval of the foreign body or tooth fragment for fibrosis to set in and stabilize in firm position. According to some authors delayed intervention may increase the risk of infection and result in a foreign body reaction or migration of the fragment [7-9]. In our case the surgical intervention was delayed for one week to stabilize the already existing primary surgical area, which than would be followed by an independent second surgical incision. Also, it may be prudent to wait for the surgical wound to settle and a fresh attempt to retrieve the fragment can be made later in case we feel it may lead to inadvertent damage to vital structures around or surgical morbidity may ensue. In case the $\mathrm{CBCT}$ reveals an optimum route from the same surgical access, then an immediate retrieval must be executed. Finally, the maintenance of accurate notes is mandatory and the patient must be fully informed of the events and complications if any.

\section{References}

1. Zeltser R, Cohen C, Casap N (2002) The implications of a broken needle in the pterygomandibular space: clinical guidelines for prevention and retrieval. Pediatr Dent 24: 153-156.

2. Prado FB, Caria PHF, Silva RF, Martins EC, Daruge E (2010) Dental broken needle migration to the skull base. A case of dental broken needle migration to the skull base: Anatomical considerations and prevention. J. Morphol Sci 27: 98-101.

3. Kamburoglu K, Kursun S, Oztas B (2010) Submandibular displacement of a mandibular third molar root during extraction:a case report. Cases J 3: 8.

4. Suer BT, Kocyigit DI, Ortakoglu K (2014) latrogenic Displacement of Impacted Mandibular Third Molar into the Pterygomandibular Space: A Case Report. OHDM $13: 179-182$

5. Jolly SS, Rattan V, Rai SK (2014) Intraoral management of displaced root into submandibular space under local anaesthesia -A case report and review of literature. The Saudi Dental Journal 26: 181-184. 
Citation: Kalia V, Kalra G, Singh G, Sharma V (2015) Localization of Broken Surgical Bur in the Submandibular Space: Its Prevention, Retrieval and the Role of Cone Beam Computed Tomography (CBCT). J Clin Case Rep 5: 636. doi:10.4172/2165-7920.1000636

6. Arasa LA, Figueiredo R, Escoda CG (2012) latrogenic displacement of third molar roots in to the sublingual space: report of 6 cases. J Oral Maxillofac Surg e107-e115.

7. Gay-Escoda C, Berini-Aytes L, Pinera-Penalva M (1993) Accidental displacement of a lower third molar. Oral Surg Oral Med Oral Pathol Oral Radiol Endod 76: 159-160.
8. Dormer BJ, Babett JA (1973) Root section in the submaxillary space. Oral Surg Oral Med Oral Pathol Oral Radiol Endod 35: 876.

9. Peterson LJ (1988) Prevention and management of surgical complications. In Contemporary Oral and Maxillofacial Surgery (1st edn.) St. Louis: CV Mos by 275-277. 